\title{
KIF11 Is Required for Spheroid Formation by Oesophageal and Colorectal Cancer Cells
}

\author{
TAKEHARU IMAI ${ }^{1,2}$, NAOHIDE OUE $^{1}$, KAZUHIRO SENTANI $^{1}$, NAOYA SAKAMOTO $^{1}$, NAOHIRO URAOKA $^{1}$, \\ HIROYUKI EGI $^{3}$, TAKAO HINOI ${ }^{3,4}$, HIDEKI OHDAN $^{3}$, KAZUHIRO YOSHIDA $^{2}$ and WATARU YASUI ${ }^{1}$ \\ ${ }^{1}$ Department of Molecular Pathology, \\ Hiroshima University Institute of Biomedical and Health Sciences, Hiroshima, Japan; \\ ${ }^{2}$ Department of Surgical Oncology, Graduate School of Medicine, Gifu University, Gifu, Japan; \\ ${ }^{3}$ Department of Gastroenterological and Transplant Surgery, Applied Life Sciences, \\ Institute of Biomedical \& Health Sciences, Hiroshima University, Hiroshima, Japan; \\ ${ }^{4}$ Department of Surgery, Institute for Clinical Research, \\ National Hospital Organization Kure Medical Center and Chu-goku Cancer Center, Kure, Japan
}

\begin{abstract}
Background: Oesophageal squamous cell carcinoma (ESCC) and colorectal cancer (CRC) are common types of human cancer. Spheroid colony formation is used to characterize cancer stem cell (CSCs). In the present study, we analyzed the significance of kinesin family 11 (KIF11 in human ESCC and CRC. Materials and Methods: Expression of KIF11 in 105 ESCC and 100 CRC cases was determined using immunohistochemistry. RNA interference was used to inhibit KIF11 expression in ESCC and CRC cell lines. Results: In total, 61 out of 105 (58\%) ESCC and 62 out of 100 (62\%) CRC cases were positive for KIF11. Expression of KIF11 was not associated with any clinicopathological characteristics. Both the number and size of spheres produced by from TE-5 ESCC cells and DLD-1 CRC cells were significantly reduced upon KIF11 siRNA transfection compared to negative control siRNA transfection. Conclusion: These results indicate that KIF11 plays an important role in CSCs of ESCC and CRC.
\end{abstract}

Gastrointestinal (GI) cancer, including oesophageal squamous cell carcinoma (ESCC), gastric cancer (GC), and colorectal cancer (CRC), are common malignancies worldwide. A variety of genetic and epigenetic alterations are associated

This article is freely accessible online.

Correspondence to: Dr. Naohide Oue, MD, Ph.D., Department of Molecular Pathology, Hiroshima University Institute of Biomedical and Health Sciences, 1-2-3 Kasumi, Minami-ku, Hiroshima 7348551, Japan. Tel: +81 822575146, Fax: +81 822575149, e-mail: naoue@hiroshima-u.ac.jp

Key Words: KIF11, spheroid, cancer stem cell, colorectal cancer, oesophageal cancer. with GI cancer, and better knowledge of the changes in gene expression that occur during gastric carcinogenesis may lead to improvements in diagnosis, treatment, and prevention (1). Genes encoding transmembrane/secretory proteins that are specifically expressed in cancer are ideal diagnostic biomarkers. Moreover, if the gene product functions in the neoplastic process, the gene is not just a potential biomarker, but may also be a therapeutic target (2).

In the past decade, cancer has been recognized as a stem cell disease (3). Cancer stem cells (CSCs) are defined as malignant cells that possess the ability to initiate tumour growth and sustain self-renewal (4). Moreover, CSCs play an important role in resistance to chemotherapy (4). Therefore, characterization of CSCs is important for establishing more effective cancer treatments. One useful method for characterizing CSCs is spheroid colony formation. We previously showed that KIF 11 and kinesin family $\mathrm{C} 1$ (KIFCl) genes are more highly expressed, by more than two-fold, in spheroid-forming cells than in the parental cells of GC cell lines (5). We also showed that KIF11 protein expression is upregulated in GC tissue samples, and that both the number and size of spheres produced by GC cells are significantly reduced by inhibition of KIF 11 (6). These results suggest that KIF11 likely plays an important role in gastric CSCs.

KIF 11 (also known as EG5) is a member of the kinesin superfamily. The kinesin superfamily proteins are classified as mitotic kinesins, which are involved in cell division, and non-mitotic kinesins, which are principally involved in intracellular transport (7). KIF 11 is a mitotic kinesin and is required for the separation of duplicated centrosomes during spindle formation (8). Thus, a KIF11 inhibitor is thought to be useful to specifically target proliferating tumour tissue (9). Several small molecule KIF11 inhibitors have been reported (10). There is a possibility that KIF 11 inhibitors 
may exhibit antitumour activity in patients with GI cancer. Despite the potential connection between KIF11 and cancer, the significance of KIF11 in ESCC and CRC has not been characterized.

In the present study, we analyzed the expression and distribution of KIF11 in human ESCC and CRC by immunohistochemistry, and examined the relationship between KIF11 expression and clinicopathological characteristics of patients with ESCC, and CRC. Furthermore, we also analyzed the effect of inhibiting KIF 11 expression by RNA interference (RNAi) on spheroid formation in ESCC and CRC cells.

\section{Materials and Methods}

Tissue samples. In a retrospective study design, 113 primary tumours were collected from patients diagnosed with ESCC and 113 primary tumours were collected from patients diagnosed with CRC who underwent surgery at Hiroshima University Hospital (Hiroshima, Japan). All patients underwent curative resection. Only patients without preoperative radiotherapy or chemotherapy and without clinical evidence of distant metastasis were enrolled in the study. Operative mortality was defined as death within 30 days of patients leaving the hospital, and these patients were removed from the analysis. Postoperative follow-up was scheduled every 1, 2 or 3 months during the first 2 years after surgery and every 6 months thereafter unless more frequent follow-up was deemed necessary. Chest X-ray, chest computed tomographic scan and serum chemistry analysis were performed at every follow-up visit. Patients were followed by their physician until death or the date of the last documented contact.

Tumour staging was determined according to the TNM classification system (11). Histological classification of classifications of ESCC and CRC were based on the World Health Organization system $(12,13)$. This study was approved (no. IRINHI66) by the Ethical Committee for Human Genome Research of Hiroshima University (Hiroshima, Japan).

Quantitative reverse transcription-polymerase chain reaction ( $q R T$ PCR) analysis. For qRT-PCR, eight ESCC samples and 13 CRC samples were examined. Samples were frozen immediately in liquid nitrogen and stored at $-80^{\circ} \mathrm{C}$ until use. Total RNA was extracted with an RNeasy Mini Kit (Qiagen, Valencia, CA, USA.), and $1 \mu \mathrm{g}$ of total RNA was converted to cDNA using First Strand cDNA Synthesis Kit (Amersham Biosciences, Piscataway, NJ, USA). Quantitation of KIFI1 mRNA level was performed by real-time fluorescence detection as described previously (6). PCR was conducted using the SYBR Green PCR Core Reagents Kit (Applied Biosystems). Real-time detection of the emission intensity of SYBR green bound to double-stranded DNA was performed with the ABI PRISM 7700 Sequence Detection System (Applied Biosystems). Actin-beta $(A C T B)$-specific PCR products were amplified from the same RNA samples and served as an internal control.

Immunohistochemistry. We used archival formalin-fixed, paraffinembedded tissues from a total of 205 patients who had undergone surgical excision either for ESCC $(n=105)$ or for CRC $(n=100)$. In addition, 20 colorectal adenoma cases obtained by endoscopic mucosal resection were collected. Immunohistochemical analysis was performed with the Dako Envision+ Mouse Peroxidase
Detection System (Dako Cytomation). Antigen retrieval was performed by microwave heating in citrate buffer $(\mathrm{pH}$ 6.0) for 30 min. Peroxidase activity was blocked with $3 \% \mathrm{H}_{2} \mathrm{O}_{2}$-methanol for $10 \mathrm{~min}$. Sections were incubated with a mouse monoclonal antibody to KIF11 (1:50, Abcam) or antibody to aldehyde dehydrogenase 1 family member A1 (ALDH1) (1:200; BD Biosciences, BD Biosciences; San Diego, CA, USA) for $1 \mathrm{~h}$ at room temperature, followed by incubation with Envision+ anti-mouse peroxidase for $1 \mathrm{~h}$. For colour reaction, sections were incubated with DAB Substrate-Chromogen Solution (Dako Cytomation, Carpinteria, CA, USA) for $10 \mathrm{~min}$. Sections were counterstained with $0.1 \%$ haematoxylin. Negative controls were created by omission of the primary antibody. Expression of KIF1 and ALDH1 was scored in all tumours as positive or negative; when more than $10 \%$ of tumour cells were stained, immunostaining was considered positive.

Sections were incubated with the following antibody dilutions; primary anti-CD133 antibody (AC133; Miltenyi Biotec, Auburn, CA, USA) 1:100, anti-ALDH1 anti-body (BD Biosciences; San Diego, CA, USA) 1:200, and anti-CD44 antibody (Novocastra; Newcastle, UK). Sections were incubated with primary antibody for $1 \mathrm{~h}$ at room tem-perature. Sections were incubated with the following antibody dilutions; primary anti-CD133 antibody (AC133; Miltenyi Biotec, Auburn, CA, USA) 1:100, anti-ALDH1 anti-body (BD Biosciences; San Diego, CA, USA) 1:200, and anti-CD44 antibody (Novocastra; Newcastle, UK). Sections were incubated with primary antibody for $1 \mathrm{~h}$ at room temperature. Sections were incubated with the following antibody dilutions; primary anti-KIF11 antibody (1:50, Abcam), anti-ALDH1 anti-body (1:200, BD Biosciences). Sections were incubated with primary antibody for 1 $\mathrm{h}$ at room temperature.

Cell lines. Four cell lines derived from human oesophageal cancer (TE-1, TE-5, TE-8, and TE-9) and five cell lines derived from human CRC (COLO320, Lovo-JC, DLD-1, CCK, and WiDr) were used. All cell lines were purchased from the Japanese Collection of Research Bioresources Cell Bank (Osaka, Japan). All cell lines were maintained in RPMI-1640 (Nissui Pharmaceutical Co, Ltd, Tokyo, Japan) containing $10 \%$ foetal bovine serum (BioWhittaker, Walkersville, MD, USA) in a humidified atmosphere of $5 \% \mathrm{CO}_{2}$ and $95 \%$ air at $37^{\circ} \mathrm{C}$.

$R N A i$. Short interfering RNA (siRNA) oligonucleotides targeting KIF11 and a negative control were purchased from Invitrogen (Carlsbad, CA, USA). We used three independent KIF11 siRNA oligonucleotide sequences. Transfection was performed using Lipofectamine RNAiMAX (Invitrogen) as described previously (14). Briefly, 60 pmol of siRNA and $10 \mu \mathrm{l}$ of Lipofectamine RNAiMAX were mixed in $1 \mathrm{ml}$ of RMPI medium (10 nmol/l final siRNA concentration). After $20 \mathrm{~min}$ of incubation, the mixture was added to cells and then cells were plated in culture dishes. Fortyeight hours after transfection, cells were analysed for spheroid colony formation.

Spheroid colony formation. For the generation of spheres, $2 \times 10^{3}$ cells (transfected with KIF11 siRNA, or negative control siRNA) were plated on 24-well ultra-low attachment plates (Corning, New York, NY, USA). Cells were grown in mTeSR medium (STEMCELL Technologies Inc., Cambridge, MA, USA). The plates were incubated at $37^{\circ} \mathrm{C}$ in a $5 \% \mathrm{CO}_{2}$ incubator for 15 days. Sphere number and size were then determined using a microscope. 
Table I. Relationship between kinesin family 11 KIF11 expression and clinicopathological characteristics in oesophageal squamous cell carcinoma .

\begin{tabular}{|c|c|c|c|c|}
\hline \multirow[b]{2}{*}{ Characteristic } & \multirow[b]{2}{*}{ Subgroup } & \multicolumn{2}{|c|}{ KIF11 expression, $\mathrm{n}(\%)$} & \multirow[b]{2}{*}{$p$-Value } \\
\hline & & Positive & Negative & \\
\hline \multirow[t]{2}{*}{ Age } & $<65$ Years & $25(56)$ & $20(44)$ & 0.6478 \\
\hline & $\geq 65$ Years & $36(60)$ & $24(40)$ & \\
\hline \multirow[t]{2}{*}{ Gender } & Male & $7(47)$ & $8(53)$ & 0.3326 \\
\hline & Female & $54(60)$ & $36(40)$ & \\
\hline \multirow[t]{2}{*}{$\mathrm{T}$ classification } & $\mathrm{T} 1$ & $26(52)$ & $24(48)$ & 0.2582 \\
\hline & $\mathrm{T} 2 / 3 / 4$ & $34(63)$ & $20(37)$ & \\
\hline \multirow[t]{2}{*}{$\mathrm{N}$ classification } & No & $22(47)$ & $25(53)$ & 0.0413 \\
\hline & $\mathrm{N} 1 / 2 / 3$ & $38(66)$ & $19(34)$ & \\
\hline \multirow[t]{2}{*}{ Stage } & I & $17(47)$ & $19(53)$ & 0.1158 \\
\hline & II/III/IV & $43(63)$ & $25(37)$ & \\
\hline \multirow[t]{2}{*}{ Lymphovascular invasion } & Negative & $39(62)$ & $24(38)$ & 0.3326 \\
\hline & Positive & $22(53)$ & $20(47)$ & \\
\hline \multirow[t]{2}{*}{ Vascular invasion } & Negative & $43(54)$ & $36(46)$ & 0.1846 \\
\hline & Positive & $18(69)$ & $8(31)$ & \\
\hline \multirow[t]{2}{*}{ Tumour differentiation } & Well or moderately & $42(55)$ & $35(45)$ & 0.2215 \\
\hline & Poorly & $19(68)$ & $9(32)$ & \\
\hline \multirow[t]{2}{*}{ Ki-67 expression $(n=100)$} & $<30 \%$ & $36(59)$ & $25(41)$ & 0.8017 \\
\hline & $\geq 30 \%$ & $24(62)$ & $15(38)$ & \\
\hline \multirow[t]{2}{*}{ ALDH1 expression } & $<10 \%$ & $29(62)$ & $18(38)$ & $<0.0001$ \\
\hline & $\geq 10 \%$ & $54(93)$ & $4(7)$ & \\
\hline
\end{tabular}

ALDH1: Aldehyde dehydrogenase 1 family member A1.

Western blot analysis. KIF11 siRNA-transfected, and nontransfected cells were lysed as described previously (15). The lysates $(40 \mu \mathrm{g})$ were solubilized in Laemmli buffer by boiling and then subjected to $10 \%$ sodium dodecyl sulphate-polyacrylamide gel electrophoresis followed by electrotransfer onto a nitrocellulose filter. Monoclonal antibody to KIF11 was purchased from Abcam (Cambridge, MA, USA). Peroxidase-conjugated anti-mouse IgG was used in the secondary reaction. Immunocomplexes were visualized with an ECL Western Blot Detection System (Amersham Biosciences). $\beta$-Actin (Sigma, St. Louis, MO, USA) was used as a loading control.

Statistical methods. Associations between clinicopathological parameters and KIF11 expression were analysed by Fisher's exact test. Kaplan-Meier survival curves were constructed for KIF11positive and KIF11-negative patients. Survival rates were compared between KIF11-positive and KIF11-negative groups. Differences between survival curves were tested for statistical significance by a log-rank test. Differences in the sphere number and size between the two groups (KIF11 siRNA-transfected cells and negative control siRNA-transfected cells) were tested by Student $t$-test.

\section{Results}

Expression of KIF11 mRNA in ESCC tissue and nonneoplastic mucosa samples. We first analyzed mRNA expression of KIF11 in eight ESCC tissue samples and nonneoplastic mucosa samples by qRT-PCR (Figure 1A). Overexpression of KIF11 (tumour/non-neoplastic mucosa ratio $>2$ ) was observed in six out of the eight ESCC tissue samples.
The expression and distribution of KIF11 protein in ESCC has not been investigated to our knowledge. Therefore, we performed immunohistochemical analysis of KIF11 in 105 ESCC tissue samples. In non-neoplastic mucosa, weak or no staining of KIF11 was observed in the epithelial cells. In contrast, ESCC tissue showed stronger, more extensive KIF11 staining than non-neoplastic mucosa (Figure 1B). Staining of KIF11 was observed mainly in the nuclei. The percentage of KIF11-stained ESCC cells in the samples ranged from $0 \%$ to $90 \%$. We classified immunohistochemical staining as KIF11-positive when more than $10 \%$ of tumour cells were stained for KIF11. In total, 61 (58\%) of 105 ESCC cases were positive for KIF11.

Next, we analyzed whether KIF11 is associated with CSCs. CSC markers include cluster of differentiation (CD)133, CD44, CD24, CD166, and ALDH1, and among these, ALDH1 is widely used (16). Therefore, we performed immunostaining of ALDH1 in 105 ESCC cases. In the ESCC samples, ALDH1 staining was observed mainly in the ESCC cells. Among the 105 ESCC cases, 54 (51\%) were positive for ALDH1. As shown in Figure 1C, expression of KIF11 was observed frequently in ALDH1-positive ESCC cells. Indeed, Fisher's exact test demonstrated that KIF11-positivity in ESCC was significantly frequently associated with ALDH1 positivity $(p<0.0001$; Table I).

We next examined the relationship of KIF11 staining to clinicopathological characteristics in ESCC cases (Table I). 
A

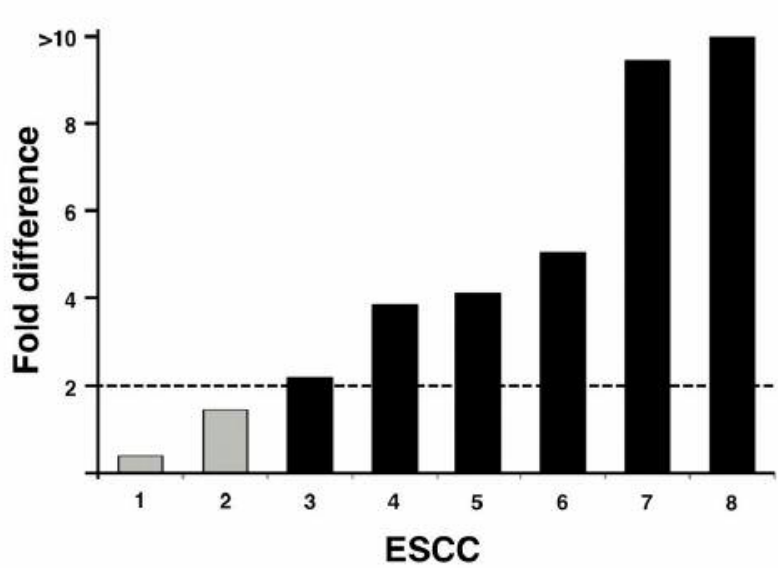

B

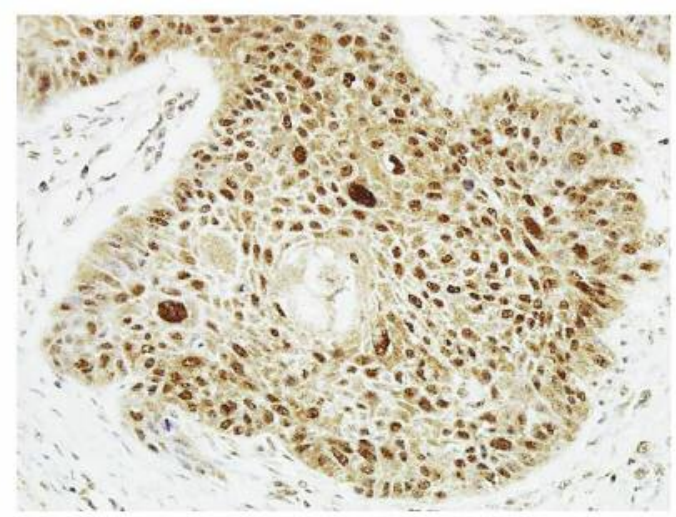

C

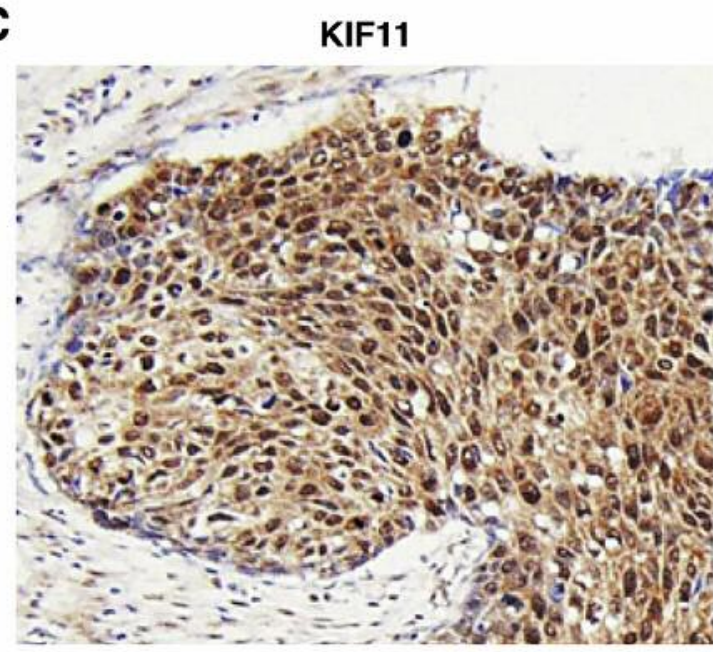

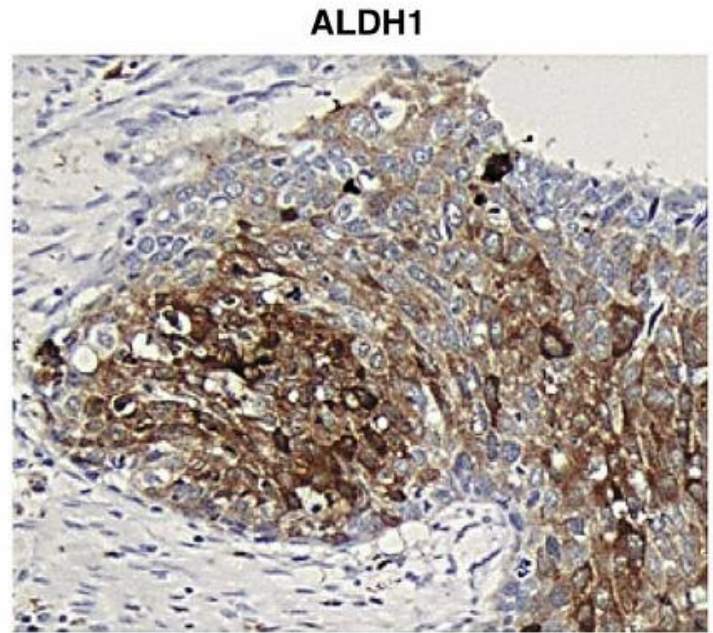

D

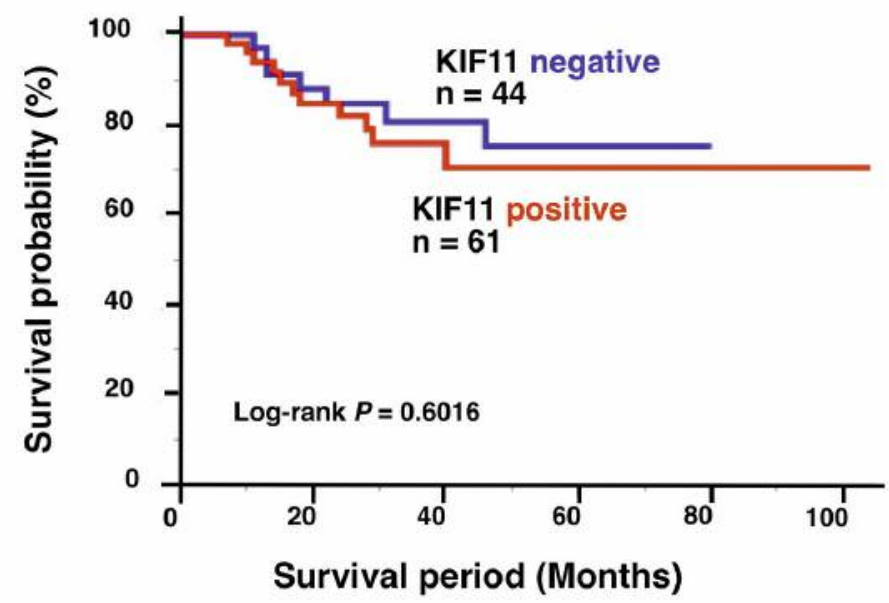

Figure 1. Expression of kinesin family 11 (KIF11) in oesophageal squamous cell carcinoma (ESCC) tissues. A: Quantitative reverse transcriptionpolymerase chain reaction analysis of KIF11 in eight ESCC samples. The bars represent individual samples. Fold difference is the ratio of KIF11 mRNA level in ESCC to that in corresponding non-neoplastic mucosa. B: Immunohistochemical analysis of KIF11 in ESCC; original magnification, $\times 400$. C: Immunohistochemical analysis of KIF11 (left) and ALDH1 (right) in consecutive tumour sections of ESCC, showing marked coexpression; original magnification, $\times 400$. D: Kaplan-Meier plot of survival for patients with ESCC by tumour KIF11 expression. 
A

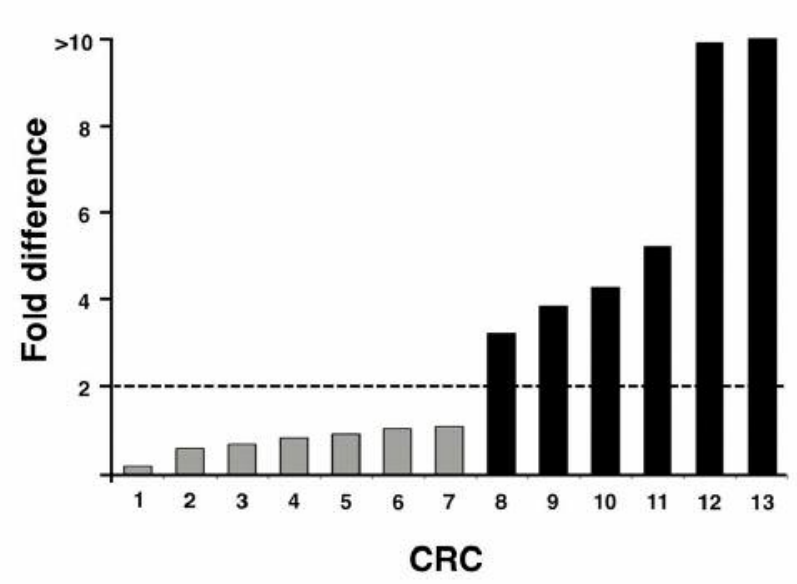

B

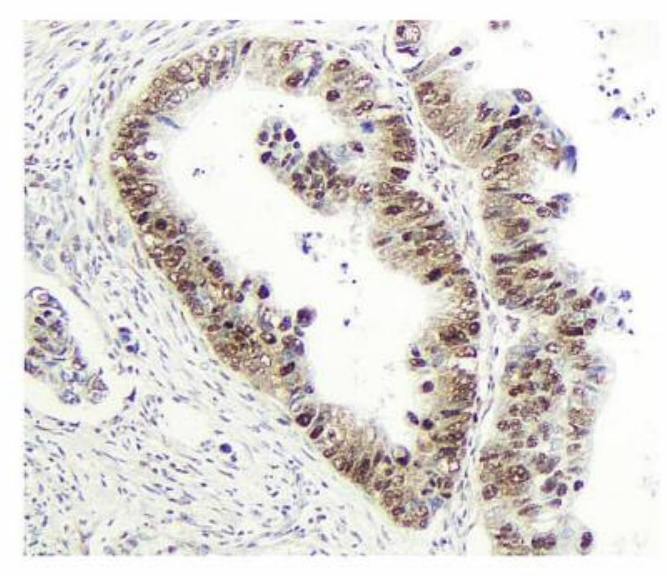

C

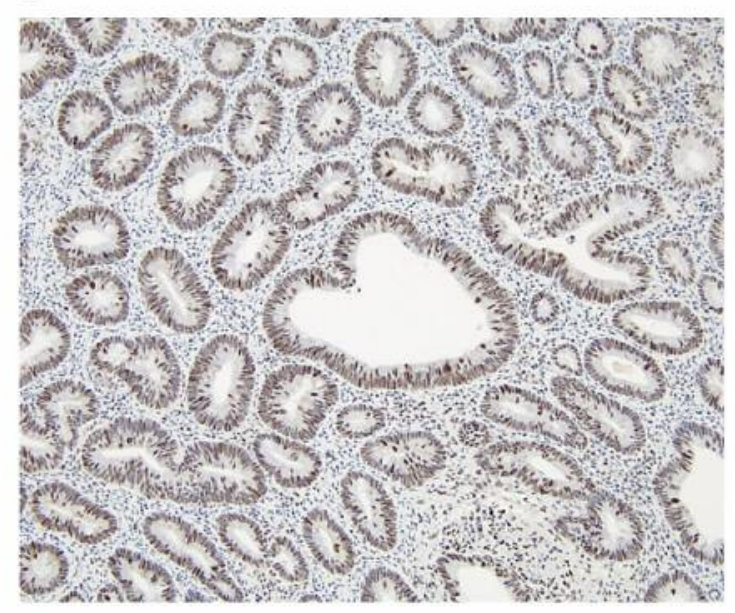

D

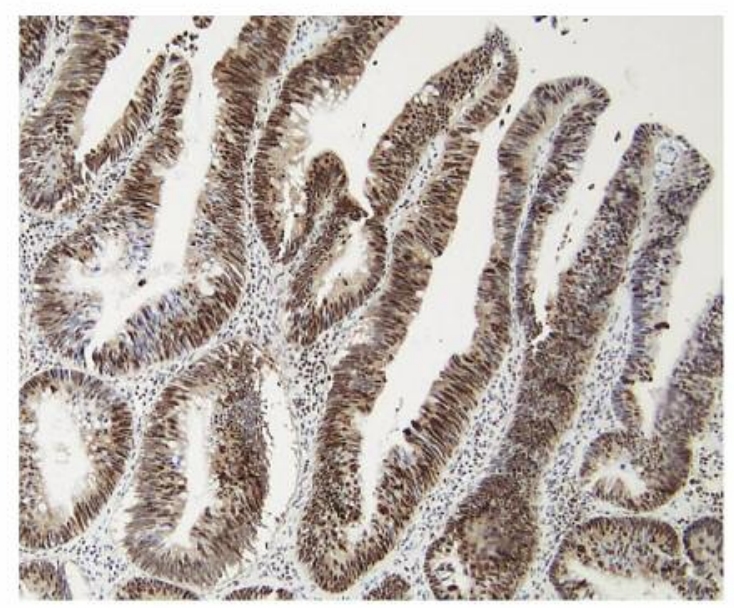

$\mathbf{E}$

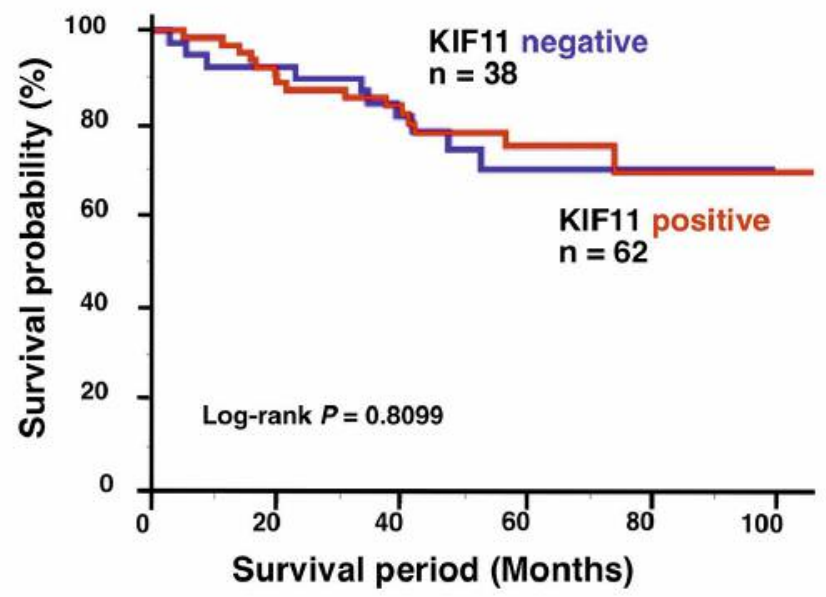

Figure 2. Expression of kinesin family 11 (KIF11) in colorectal cancer (CRC) tissues. A: Quantitative reverse transcription-polymerase chain reaction analysis of KIF11 in 13 CRC samples. The bars represent individual samples. Fold difference is the ratio of KIF11 mRNA level in CRC to that in corresponding non-neoplastic mucosa. B: Immunohistochemical analysis of KIF11 in CRC; original magnification, $\times 400$. Immunohistochemical analysis of KIF11 in low-grade $(C)$ and high-grade $(D)$ adenoma; original magnification, $\times 100$. E: Kaplan-Meier plot of survival in patients with CRC by tumour KIF11 expression. 
A
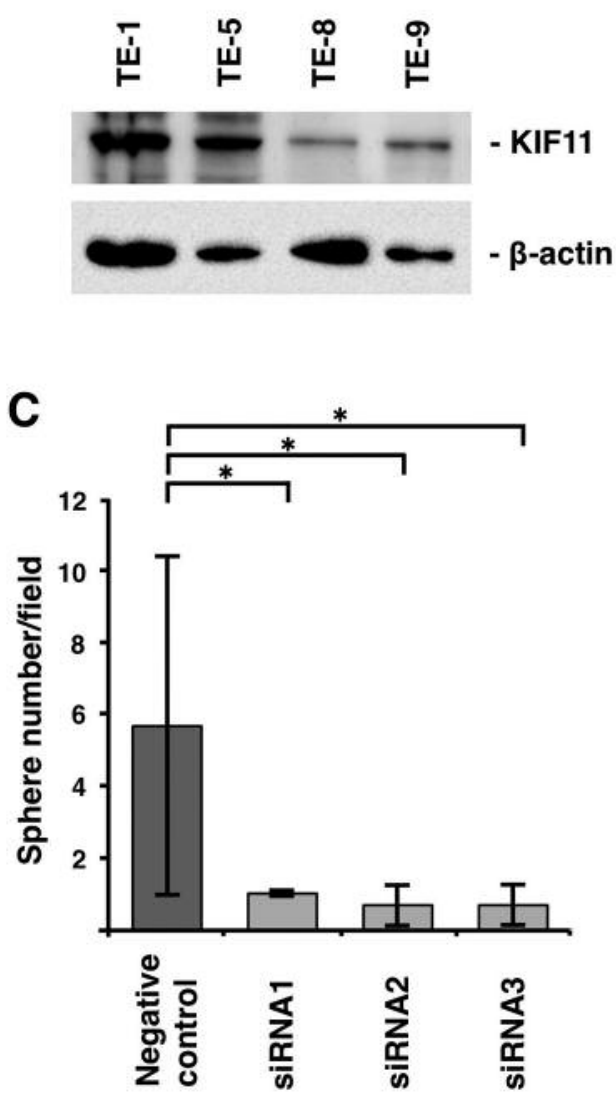

B
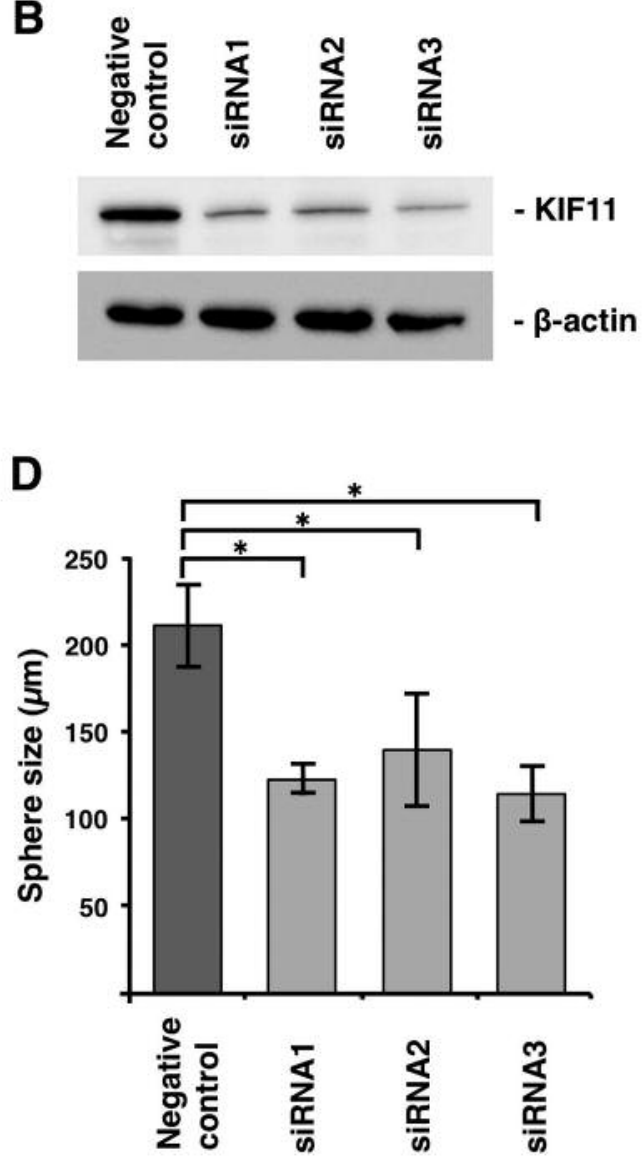

Figure 3. Effect of kinesin family 11 (KIF11) inhibition in oesophageal squamous cell carcinoma (ESCC) cells. A: Western blot analysis of KIF11 in four ESCC cell lines. B: Western blot analysis of KIF11 in cell lysates from TE-5 cells transfected with KIF11 siRNA or negative control siRNA. $\beta$-Actin was included as a loading control. Number $(C)$ and size $(D)$ of spheres produced by TE-5 ESCC cells transfected with KIF11 siRNA or negative control siRNA. Forty-eight hours after transfection, cells were incubated at $37^{\circ} \mathrm{C}$ in a $5 \% \mathrm{CO}_{2}$ incubator for 15 days for spheroid colony formation. Bars and error bars indicate the mean and SD, respectively, of three experiments. *Significantly different at p<0.05.

Expression of KIF11 was associated with N classification. Kaplan-Meier analysis demonstrated that KIF11 expression was not significantly associated with survival (Figure 1D). Univariate and multivariate Cox proportional hazards analysis also showed that KIF11 expression was not a prognostic predictor for survival in patients with ESCC (data not shown). These results suggest that KIF11 plays an important role in the pathogenesis of ESCC, but not its progression.

Expression of KIF11 mRNA in CRC tissue and nonneoplastic mucosa samples. We also analyzed expression of KIF11 in CRC. We first performed qRT-PCR analysis of KIF 11 mRNA in 13 CRC tissue samples and non-plastic tissue samples (Figure 2A). Overexpression of KIF11 (tumour/non-neoplastic mucosa ratio $>2$ ) was detected in six out of $13(46 \%)$ CRC tissue samples.
The expression and distribution of KIF11 protein in CRC has also not been investigated as far as we are aware. Therefore, we performed immunohistochemical analysis of KIF11 in 100 CRC tissue samples. In non-neoplastic mucosa, weak or no staining of KIF11 was observed in the epithelial cells. In contrast, CRC tissue showed stronger, more extensive staining than non-neoplastic mucosa (Figure 2B). KIF11 staining was observed mainly in nuclei. In total, 62 out of 100 (62\%) CRC cases were positive for KIF11.

We also performed immunohistochemical analysis of KIF11 in 10 low-grade (Figure 2C) and 10 high-grade (Figure 2D) colorectal adenoma cases. In total, eight out of 10 low-grade and nine out of 10 high-grade adenoma cases were positive for KIF11. These results suggest that overexpression of KIF11 is an early event in the pathogenesis of CRC. 
A

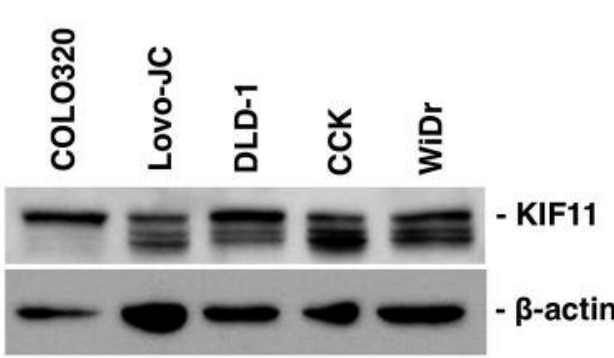

C

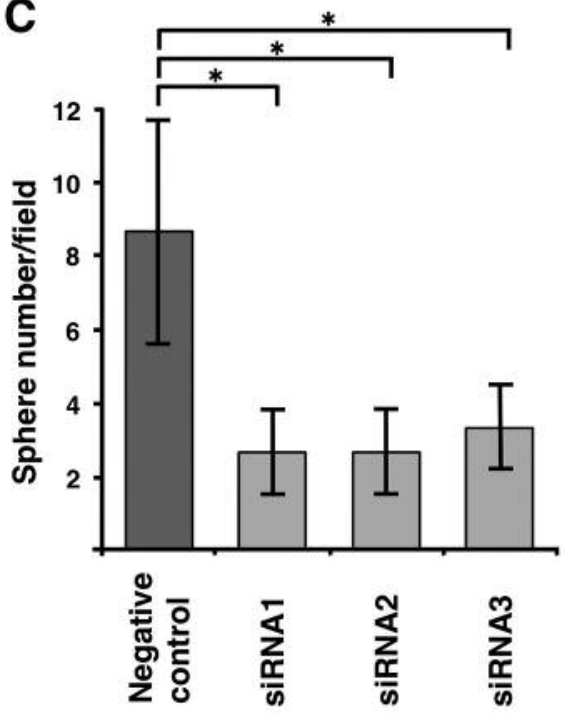

B
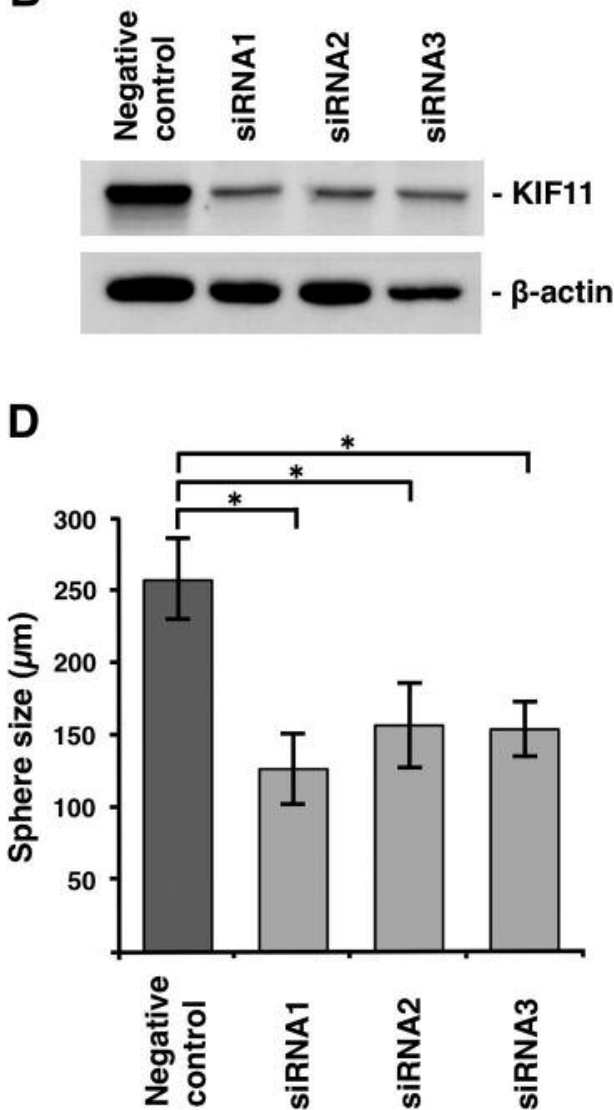

Figure 4. Effect of kinesin family 11 (KIF11) inhibition in colorectal cancer (CRC) cells. A. Western blot analysis of KIF11 in five CRC cell lines. B: Western blot analysis of KIF11 in cell lysates from DLD-1 cells transfected with KIF11 siRNA or negative control siRNA. $\beta$-Actin was included as a loading control. Number $(C)$ and size $(D)$ of spheres produced by produced by DLD-1 CRC cells transfected with KIF11 siRNA or negative control siRNA. Forty-eight hours after transfection, cells were incubated at $37^{\circ} \mathrm{C}$ in a $5 \% \mathrm{CO}_{2}$ incubator for 15 days for spheroid colony formation. Bars and error bars indicate the mean and SD, respectively, of three different experiments. *Significantly different at $p<0.05$.

We next examined the relationship of KIF11 staining to clinicopathological characteristics (Table II). Expression of KIF11 was associated with T classification and histological classification. Kaplan-Meier analysis demonstrated that KIF11 expression was not significantly associated with survival (Figure 2E). Univariate and multivariate Cox proportional hazards analysis demonstrated that KIF11 expression was not a prognostic predictor for survival in patients with CRC (data not shown).

Effect of inhibition of KIF11 on sphere number and size. Our findings demonstrated that KIF 11 was up-regulated in ESCC and CRC tissues. However, the significance of KIF 11 expression in oesophageal and colorectal CSC remains unclear. Therefore, we examined the effect of KIF11 inhibition on sphere number and size.
We first performed western blot analysis of KIF11 in four ESCC cell lines and confirmed that the antibody to KIF11 detected a band of approximately $120 \mathrm{kDa}$ in all ESCC cell lines (Figure 3A). We next analysed the effect of three siRNAs targeting KIF11 (siRNA1, siRNA2, and siRNA3) by transfecting each siRNA into TE-5 cells, and confirmed that the expression of endogenous KIF11 was substantially suppressed by all three siRNAs (Figure 3B). Therefore, we used siRNA1, siRNA2, and siRNA3 in subsequent experiments to knock-down endogenous KIF11 expression. We analyzed sphere number and size in TE- 5 cells 15 days after siRNA transfection. The number of spheres was significantly reduced in KIF 11 siRNA-transfected TE-5 cells compared with the negative control siRNA-transfected cells (Figure 3C). The size of spheres was also significantly reduced in KIF11 siRNA-transfected TE-5 cells compared 
Table II. Relationship between kinesin family 11 KIF11 expression and clinicopathological characteristics in colorectal cancer.

\begin{tabular}{|c|c|c|c|c|}
\hline \multirow[b]{2}{*}{ Characteristic } & \multirow[b]{2}{*}{ Subgroup } & \multicolumn{2}{|c|}{ KIF11 expression, $\mathrm{n}(\%)$} & \multirow[b]{2}{*}{$p$-Value } \\
\hline & & Positive (\%) & Negative & \\
\hline \multirow[t]{2}{*}{ Age } & $<65$ Years & $31(60)$ & $21(40)$ & 0.6091 \\
\hline & $\geq 65$ Years & $31(65)$ & $17(35)$ & \\
\hline \multirow[t]{2}{*}{ Gender } & Male & $41(67)$ & $20(33)$ & 0.1792 \\
\hline & Female & $21(54)$ & $18(46)$ & \\
\hline \multirow[t]{2}{*}{$\mathrm{T}$ Classification } & $\mathrm{T} 1$ & $3(27)$ & $8(73)$ & 0.0119 \\
\hline & $\mathrm{T} 2 / 3 / 4$ & $59(66)$ & $30(34)$ & \\
\hline \multirow[t]{2}{*}{ N Classification } & No & $32(58)$ & $23(42)$ & 0.3845 \\
\hline & $\mathrm{N} 1 / 2 / 3$ & $30(67)$ & $15(33)$ & \\
\hline \multirow[t]{2}{*}{ Stage } & I & $14(52)$ & $13(48)$ & 0.2035 \\
\hline & II/III/IV & $48(66)$ & $25(34)$ & \\
\hline \multirow[t]{2}{*}{ Tumour location } & Right colon & $10(59)$ & $7(41)$ & 0.7671 \\
\hline & Left colon & $52(63)$ & $31(37)$ & \\
\hline \multirow[t]{2}{*}{ Tumour differentiation } & Well or moderately & $62(65)$ & $34(35)$ & 0.0091 \\
\hline & Other & $0(0)$ & $4(100)$ & \\
\hline
\end{tabular}

with the negative control siRNA-transfected cells (Figure 3D). Similar results were obtained using TE-1 cells (data not shown).

We performed similar experiments in CRC cell lines. We first detected KIF11 expression in five CRC cell lines by western blot analysis (Figure 4A) and confirmed that the three siRNAs were able to effectively knock-down KIF11 in DLD-1 CRC cells (Figure 4B). We analyzed sphere formation in DLD-1 cells knocked-down for KIF 11 and found that the number and size of spheres was significantly reduced in KIF 11 siRNA-transfected DLD-1 cells compared to negative control siRNA-transfected cells (Figure 4C and D). These results suggest that KIFI1 is indeed required for sphere formation in ESCC and CRC cells.

\section{Discussion}

Previously, we reported that KIF11 protein expression is upregulated in $72 \%$ of GC cases and that KIF11 is involved in spheroid formation (6). In the present study, we analyzed KIF11 expression in ESCC and CRC tissues. Although weak or no staining of KIF11 was observed in non-neoplastic oesophageal mucosa and colorectal mucosa, 58\% of ESCC cases and $61 \%$ of CRC cases were positive for KIF11 by immunohistochemistry. Furthermore, expression of KIF11 was not associated with TNM stage or patient survival. Taken together, these results indicate that KIF11 plays an important role in the pathogenesis of ESCC and CRC but not its progression.

We previously demonstrated that KIFI1 and KIFC1 genes are more highly expressed in spheroid-forming cells than in parental GC cells (5). We also reported that KIF11 likely plays an important role in gastric CSCs (6). In the present study, we found that both the number and size of spheres produced by ESCC and CRC cell lines were significantly reduced by KIFI1 siRNA transfection compared with negative control siRNA-transfected cells, indicating that KIF11 is required for sphere formation of ESCC and CRC cells. Moreover, we found that KIF11-positivity in ESCC was significantly frequently associated with ALDH1 positivity by immunohistochemistry. Taken together, these results suggest that $K I F 11$ is likely to help in the production of CSC in ESCC and CRC.

Mitotic kinesins such as KIF11 are involved in cell division and non-mitotic kinesins are principally involved in intracellular transport. KIF11 is required for the separation of duplicated centrosomes during spindle formation (8). KIF11 inhibitors have entered phase 1 and 2 clinical trials either as monotherapies or in combination with other drugs (7). KIF11 is targeted by one of the most advanced mitotic kinesin inhibitors, filanesib (also known as ARRY-520). Filanesib is a highly selective, targeted inhibitor of KIF 11 that induces mitotic arrest and subsequent tumour cell death. A first-in-human phase 1 study in patients with advanced solid tumours has been completed and the results concluded that filanesib provides exposure with acceptable tolerability and evidence of target-specific pharmacodynamic effects (17). In our study, 58\% of ESCC cases and $61 \%$ of CRC cases were positive for KIF11 by immunohistochemistry, indicating that KIF 11 inhibitors, such as filanesib, might be effective in patients with ESCC or CRC. KIF 11 inhibition leads to the activation of the spindle checkpoint, mitotic arrest and subsequent cell death in certain cancer cell lines (17). Inhibition of KIF11 was found to stop the growth of 
treatment-resistant glioblastoma tumour-initiating cells (18). Therefore, inhibition of KIF11 may have positive effects in treatment-resistant ESCC or CRC.

In summary, we found that KIFI1 is overexpressed in ESCC and CRC. KIF11 likely plays an important role in ESCC and CRC. Furthermore, our results showed that knockdown of KIF11 by siRNA inhibits sphere formation, indicating that KIFII is important in activity of oesophageal and colorectal CSCs.

\section{Conflicts of Interest}

The Authors declare no conflicts of interest.

\section{Acknowledgements}

This work was supported by Grants-in-Aid for Scientific Research (B) $(15 \mathrm{H} 04713)$ and for Challenging Exploratory Research (26670175, 16K15247) from the Japan Society for the Promotion of Science.

\section{References}

1 Oue N, Sentani K, Sakamoto N and Yasui W: Clinicopathologic and molecular characteristics of gastric cancer showing gastric and intestinal mucin phenotype. Cancer Sci 106: 951-958, 2015

2 Oue N, Naito Y, Hayashi T, Takigahira M, Kawano-Nagatsuma A, Sentani K, Sakamoto N, Zarni Oo H, Uraoka N, Yanagihara $\mathrm{K}$, Ochiai A, Sasaki H and Yasui W: Signal peptidase complex 18 , encoded by $S E C 11 A$, contributes to progression via TGFalpha secretion in gastric cancer. Oncogene 33: 3918-3926, 2014.

3 Bessede E, Dubus P, Megraud F and Varon C: Helicobacter pylori infection and stem cells at the origin of gastric cancer. Oncogene 34: 2547-2555, 2015.

4 Takaishi S, Okumura T and Wang TC: Gastric cancer stem cells. J Clin Oncol 26: 2876-2882, 2008.

5 Oue N, Mukai S, Imai T, Pham TT, Oshima T, Sentani K, Sakamoto $\mathrm{N}$, Yoshida $\mathrm{K}$ and Yasui W: Induction of KIFC1 expression in gastric cancer spheroids. Oncol Rep 36: 349-355, 2016.

6 Imai T, Oue N, Nishioka M, Mukai S, Oshima T, Sakamoto N, Sentani K, Matsusaki K, Yoshida $\mathrm{K}$ and Yasui W: Overexpression of KIF11 in gastric cancer with intestinal mucin phenotype. Pathobiology 84: 16-24, 2017.

7 Rath O and Kozielski F: Kinesins and cancer. Nat Rev Cancer 12: 527-539, 2012.

8 Zhu C1, Zhao J, Bibikova M, Leverson JD, Bossy-Wetzel E, Fan JB, Abraham RT and Jiang W: Functional analysis of human microtubule-based motor proteins, the kinesins and dyneins, in mitosis/cytokinesis using RNA interference. Mol Biol Cell 16: 3187-3199, 2005.
9 Peters T, Lindenmaier H, Haefeli WE and Weiss J: Interaction of the mitotic kinesin EG5 inhibitor monastrol with P-glycoprotein. Naunyn Schmiedebergs Arch Pharmacol 372: 291-299, 2006.

10 Nakai R, Iida S, Takahashi T, Tsujita T, Okamoto S, Takada C, Akasaka K, Ichikawa S, Ishida $\mathrm{H}$, Kusaka H, Akinaga S, Murakata C, Honda S, Nitta M, Saya H and Yamashita Y: K858, a novel inhibitor of mitotic kinesin EG5 and antitumor agent, induces cell death in cancer cells. Cancer Res 69: 3901-3909, 2009.

11 Sobin LH, Gospodarowicz MK and Wittekind CH (eds.): TNM Classification of Malignant Tumors, Seventh Edition. New York, Wiley-Liss, pp 63-135, 2009.

12 Bosman FT, Carneiro F, Hruban and Theise ND (eds.): WHO Classification of Tumours of the Digestive System, Fourth Edition. Lyon, IARC, p16, 2010.

13 Bosman FT, Carneiro F, Hruban RH and Theise ND (eds.): WHO Classification of Tumours of the Digestive System, Fourth Edition. Lyon, IARC, pp. 132, 2010.

14 Sakamoto N, Oue N, Sentani K, Anami K, Uraoka N, Naito Y, Oo HZ, Hinoi T, Ohdan H, Yanagihara K, Aoyagi K, Sasaki H and Yasui W: Liver-intestine cadherin induction by epidermal growth factor receptor is associated with intestinal differentiation of gastric cancer. Cancer Sci 103: 1744-1750, 2012.

15 Yasui W, Ayhan A, Kitadai Y, Nishimura K, Yokozaki H, Ito H and Tahara E: Increased expression of $\mathrm{p} 34 \mathrm{CDC} 2$ and its kinase activity in human gastric and colonic carcinomas. Int $\mathrm{J}$ Cancer 53: 36-41, 1993.

16 Tomita H, Tanaka K, Tanaka $\mathrm{T}$ and Hara A: Aldehyde dehydrogenase 1A1 in stem cells and cancer. Oncotarget 7: 11018-11032, 2016.

17 LoRusso PM, Goncalves PH, Casetta L, Carter JA, Litwiler K, Roseberry D, Rush S, Schreiber J, Simmons HM, Ptaszynski M and Sausville EA: First-in-human phase 1 study of filanesib (ARRY-520), a kinesin spindle protein inhibitor, in patients with advanced solid tumors. Invest New Drugs 33: 440-449, 2015.

18 Venere M, Horbinski C, Crish JF, Jin X, Vasanji A, Major J, Burrows AC, Chang C, Prokop J, Wu Q, Sims PA, Canoll P, Summers MK, Rosenfeld SS and Rich JN: The mitotic kinesin KIF11 is a driver of invasion, proliferation, and self-renewal in glioblastoma. Sci Trans1 7: 304ra143, 2015.
Received November 1, 2016

Revised November 25, 2016

Accepted November 29, 2016 